\title{
DERECHO Y POLÍTICAS AMBIENTALES EN LA COMUNIDAD DE MADRID
}

(SEGUNDO SEMESTRE 2019)

\author{
Daniel B. Entrena Ruiz \\ Profesor Contratado Doctor \\ Universidad Carlos III de Madrid
}


Sumario: 1. Decreto 73/2019, de 27 de agosto, del Consejo de Gobierno, por el que se modifica la estructura orgánica básica de las consejerías de la comunidad de Madrid. 2. Resolución de 16 de julio de 2019, de la Dirección General de Medio ambiente y Sostenibilidad, por la que se aprueban los mapas estratégicos de ruido de la red de carreteras de la comunidad de Madrid. 3. Decreto 239/2019, de 24 de septiembre, del Consejo de Gobierno, por el que se declara zona de actuación urgente los terrenos afectados por el incendio ocurrido en los términos municipales de Cadalso de los Vidrios, Cenicientos y Rozas de Puerto Real. 4. Orden 1667/2019, de 2 de agosto, de la Consejería de medio ambiente y ordenación del territorio, por la que se fijan las limitaciones y épocas hábiles de caza que regirán durante la temporada 2019-2020. 5. Resolución de 10 de abril de 2019, de la Dirección General del medio ambiente y sostenibilidad, por la que se establecen la relación no exhaustiva de tramos y masas de agua aptos para las sueltas de trucha arcoíris y se establecen protocolos para llevar a cabo las mismas. 6. Orden 1501/2019, de 18 de julio, por la que se modifica la Orden 2157/2013, de 23 de septiembre, del Consejero de medio ambiente y ordenación del territorio, por la que se establecen las bases reguladoras para la concesión de las ayudas para la adquisición de vehículos eficientes para uso de autotaxi. 7. Acuerdo de la Junta de Gobierno Local del Ayuntamiento de Madrid de fecha 27 de junio de 2019, por el que "previa avocación de competencias, se establece un período de aviso en relación con el acceso a la zona de bajas emisiones Madrid Central.

\section{DECRETO 73/2019, DE 27 DE AGOSTO, DEL CONSEJO DE GOBIERNO, POR EL QUE SE MODIFICA LA ESTRUCTURA ORGÁNICA BÁSICA DE LAS CONSEJERÍAS DE LA COMUNIDAD DE MADRID}

El primer acuerdo que deseamos traer a estas páginas es el Decreto mediante el que se modifica la estructura orgánica básica de las consejerías de la Comunidad de Madrid, que expresa el planteamiento de la política ambiental que, al menos desde un punto de vista organizativo, posee el Gobierno surgido tras las Elecciones Autonómicas celebradas en mayo de 2019.

Mediante Decreto 52/2019, de 19 de agosto, la Presidenta estableció el número y denominación de las Consejerías de la Comunidad de Madrid, creando la Consejería de Medio Ambiente, Ordenación del Territorio y Sostenibilidad, que sustituye a la Consejería de Medio Ambiente, Administración Local y Ordenación del Territorio, existente hasta entonces.

De este modo, parece reforzarse el área de medio ambiente, a la que junto a la Ordenación del territorio, se le añade la Sostenibilidad, lo que parece indicar una apuesta por la perspectiva transversal del medio ambiente en el resto de políticas públicas.

El Decreto suprime varios órganos superiores y directivos, aunque parece que de forma más bien nominal, pues los cambios se limitan a añadir la mencionada Sostenibilidad. Así, se suprimen la Viceconsejería de Medio 
Ambiente y Ordenación del Territorio y la Secretaría General Técnica de Medio Ambiente y Ordenación del Territorio, y se sustituyen por la Viceconsejería de Medio Ambiente, Ordenación del Territorio y Sostenibilidad y la Secretaría General Técnica de Medio Ambiente, Ordenación del Territorio y Sostenibilidad. No obstante, ese cariz de la Sostenibilidad parece ir más allá del mero cambio nominal al suprimir la Dirección General de Medio Ambiente y Sostenibilidad, el Comisionado del Gobierno para el Cambio Climático y Agenda 2030 para el Desarrollo Sostenible, la Dirección de Área de Residuos, Calidad Ambiental y Economía Circular, y crear:

i) la Dirección General de Biodiversidad y Recursos Naturales, "que asume las competencias en materia de recursos naturales, de espacios protegidos y de análisis y coordinación que correspondían a la extinta Dirección General de Medio Ambiente y Sostenibilidad;

ii) la Dirección General de Sostenibilidad y Cambio Climático, que asume las competencias que correspondían al Comisionado del Gobierno para el Cambio Climático y Agenda 2030 para el Desarrollo Sostenible, así como las que, en materia de impacto ambiental, evaluación ambiental estratégica, desarrollo sostenible y calidad del aire, correspondían a la extinta Dirección General de Medio Ambiente y Sostenibilidad.

iii) la Dirección General de Economía Circular, que asume las competencias que correspondían a la extinta Dirección de Área de Residuos, Calidad Ambiental y Economía Circular y las de inspección y disciplina de la extinta Dirección General de Medio Ambiente y Sostenibilidad, excepto en materia de calidad del aire.

iv) Por último, se crean la Dirección General de Urbanismo, que asume las competencias que en materia de urbanismo correspondían a la extinta Dirección General de Urbanismo y Suelo, y la Dirección General de Suelo, que asume las competencias que en materia de suelo y consorcios urbanísticos correspondían a la extinta Dirección de Área de Suelo y Consorcios Urbanísticos, y se crea el Comisionado del Gobierno de Bienestar Animal.

De este modo, la Consejería de Medio Ambiente, Ordenación del Territorio y Sostenibilidad, tiene la siguiente estructura básica: 
1. Viceconsejería de Medio Ambiente, Ordenación del Territorio y Sostenibilidad, a la que se adscriben:

a) Dirección General de Biodiversidad y Recursos Naturales.

b) Dirección General de Sostenibilidad y Cambio Climático.

c) Dirección General de Economía Circular.

d) Dirección General de Agricultura, Ganadería y Alimentación.

e) Dirección General de Urbanismo.

f) Dirección General de Suelo.

2. Secretaría General Técnica.

3. Comisionado del Gobierno de Bienestar Animal.

A los anteriores órganos se añade la administración institucional adscrita a la Consejería de Medio Ambiente, Administración Local y Ordenación del Territorio, que no reproducimos por su escasa relevancia para la temática ambiental de la Revista.

\section{RESOLUCIÓN DE 16 DE JULIO DE 2019, DE LA DIRECCIÓN GENERAL} DE MEDIO AMBIENTE Y SOSTENIBILIDAD, POR LA QUE SE APRUEBAN LOS MAPAS ESTRATÉGICOS DE RUIDO DE LA RED DE CARRETERAS DE LA COMUNIDAD DE MADRID

La resolución en cuestión responde a la obligación fijada por la Directiva 2002/49/CE del Parlamento Europeo y del Consejo, de 25 de julio de 2002, sobre evaluación y gestión del ruido ambiental, de determinar por todos los países miembros la exposición al ruido ambiental mediante la elaboración de mapas estratégicos de ruido según métodos de evaluación comunes, y adoptar los correspondientes planes de acción.

Una obligación que incorpora y desarrollan la Ley 37/2003, de 17 de noviembre, del Ruido, el Real Decreto 1513/2005, de 16 de diciembre, de Evaluación y Gestión del Ruido Medioambiental, y el Real Decreto 1367/2007, de 19 de octubre, de desarrollo de la Ley 37/2003, en lo referente a zonificación acústica, objetivos de calidad y emisiones acústicas.

Este cuadro normativo fija la obligación de elaborar mapas estratégicos de ruido correspondientes a cada uno de los grandes ejes viarios cuyo tráfico supere los 3.000.000 de vehículos/año, correspondiendo dicha obligación a las 
Comunidades Autónomas respecto infraestructuras viarias de su ámbito competencial.

De este modo, tras el pertinente trámite de información pública, la Dirección General de Carreteras e Infraestructuras de la Consejería de Transportes, Vivienda e Infraestructuras de la Comunidad de Madrid elevó los mapas estratégicos de ruido de la Red de Carreteras de la Comunidad de Madrid de las vías cuyo tráfico supera los 3.000 .000 de vehículos por año, a la Consejería de Medio Ambiente, Ordenación del Territorio y Sostenibilidad, que finalmente los aprobó con fecha 16 de julio de 2009, siendo la siguiente actuación a esperar, la aprobación de los correspondientes planes de acción.

\section{DECRETO 239/2019, DE 24 DE SEPTIEMBRE, DEL CONSEJO DE} GOBIERNO, POR EL QUE SE DECLARA ZONA DE ACTUACIÓN URGENTE LOS TERRENOS AFECTADOS POR EL INCENDIO OCURRIDO EN LOS TÉRMINOS MUNICIPALES DE CADALSO DE LOS VIDRIOS, CENICIENTOS Y ROZAS DE PUERTO REAL

El Decreto del que damos noticia ahora responde a la siempre lastimosa producción de un incendio forestal, que se inició el día 28 de junio de 2019, en la localidad de Almorox (Toledo), y afectó a las localidades del oeste madrileño de Cenicientos, Cadalso de los Vidrios y Rozas de Puerto Real. Según indica el propio Decreto resultó una superficie afectada de 2.186 hectáreas en la Comunidad de Madrid, 1.711 de ellas de superficie forestal, de las que 1.461 son de titularidad privada y 250 son de titularidad pública. El del $87 \%$ perteneciente a la primera de esas localidades, y el $12 \%$ y $1 \%$, respectivamente, a las otras dos.

Por ello, para paliar sus devastadores efectos, se declara la zona afectada de Actuación Urgente, contemplando diversas medidas de restauración que hace recaer sobre los propietarios pero que asume en parte la Comunidad de Madrid, todo ello de conformidad con Ley 43/2003, de 21 de noviembre, de Montes, y la Ley 16/1995, de 4 de mayo, Forestal y de Protección de la Naturaleza. 
De esta manera se contempla, mediante el correspondiente concurso público para los montes públicos, el apeo de biomasa y masa arbolada quemada, la atuaciones de defensa del suelo frente a la erosión para evitar la pérdida de suelo, respecto las infraestructuras dañadas, zonas de refugio, agua y alimentación para la fauna silvestre y protegida de estos espacios Red Natura 2000 y control de plagas forestales (colocación de cajas nido, trampas de escolítidos, etcétera).

Respecto los montes de titularidad privada, el Decreto contempla la obligación de los titulares de los terrenos forestales afectados a iniciar las acciones restauradoras, salvo que, "en un plazo de quince días a contar desde la publicación en el BOLETÍN OFICIAL DE LA COMUNIDAD DE MADRID del Decreto, comuniquen a la Dirección General de Biodiversidad y Recursos Naturales su conformidad a convenir con la Comunidad de Madrid la realización de las actuaciones previstas en esta declaración por parte de la misma.

De esta manera, en caso de no ejecutarse en el mencionado plazo, y de conformidad con lo establecido en el artículo 72.1 de la Ley 16/1995, de 4 de mayo, Forestal y de Protección de la Naturaleza de la Comunidad de Madrid, la Administración puede optar por la ejecución subsidiaria de los trabajos, formalizar convenio forzoso o hacer uso de medidas expropiatorias.

\section{ORDEN 1667/2019, DE 2 DE AGOSTO, DE LA CONSEJERÍA DE MEDIO} AMBIENTE Y ORDENACIÓN DEL TERRITORIO, POR LA QUE SE FIJAN LAS LIMITACIONES Y ÉPOCAS HÁBILES DE CAZA QUE REGIRÁN DURANTE LA TEMPORADA 2019-2020

Mediante esta Orden se fijan las épocas hábiles de caza y sus limitaciones para la temporada 2019/2020, de conformidad con el régimen estipulado en la Ley de 4 de abril de 1970 de Caza, la Ley 42/2007, de 13 de diciembre, del Patrimonio Natural y de la Biodiversidad y la Ley 2/1991, de 14 de febrero, para la Protección y Regulación de la Fauna y Flora Silvestres de la Comunidad de Madrid. 
La Orden identifica las especies cinegéticas de caza mayor y menor que pueden ser abatidas hasta el 31 de marzo de 2020, último día del periodo regulado (aunque las fechas de caza se concretan posteriormente para caza mayor y menor y las respectivas especies), y siempre que se haya presentado previamente el correspondiente Plan de Aprovechamiento Cinegético para cada coto, de conformidad con el régimen estipulado en el Decreto 47/1991, de 21 de junio, por el que se regula la implantación obligatoria del Plan de Aprovechamiento Cinegético en los terrenos acotados en la Comunidad de Madrid.

El Decreto regula también las modalidades de caza admisibles en atención a los instrumentos y armas utilizadas, los requisitos necesarios para emplearlas, las que se encuentran prohibidas para practicar la actividad, el régimen de los animales que se empleen y las medidas de seguridad, sanitarias y de protección ambiental en las cacerías que se organicen. Finalmente, antes de concluir el referido periodo debe presentarse a la Consejería de Medio Ambiente, Ordenación del Territorio y Sostenibilidad, un informe de los resultados cinegéticos obtenidos.

\section{RESOLUCIÓN DE 10 DE ABRIL DE 2019, DE LA DIRECCIÓN GENERAL} DEL MEDIO AMBIENTE $Y$ SOSTENIBILIDAD, POR LA QUE SE ESTABLECEN LA RELACIÓN NO EXHAUSTIVA DE TRAMOS Y MASAS DE AGUA APTOS PARA LAS SUELTAS DE TRUCHA ARCOÍRIS Y SE ESTABLECEN PROTOCOLOS PARA LLEVAR A CABO LAS MISMAS

Tras regular las épocas y limitaciones de la pesca en ríos de la Comunidad de Madrid (Orden 156/2019, de 26 de febrero, de la Consejería de Medio Ambiente y Ordenación del Territorio, sobre establecimiento de vedas y regulación especial de la actividad piscícola en los ríos, arroyos y embalses de la Comunidad de Madrid), mediante esta Resolución se regula la suelta de truchas arcoriris y los protocolos para hacerlo en concretos tramos y masas de agua, y ello debido a varios motivos. 
En primer lugar, parte la Resolución de que hay un problema de conservación de las especies autóctonas ante la alta demanda de la actividad de pesca deportiva y el número de tramos en que está habilitada.

Así en el año 2018 en la Comunidad de Madrid había un total de 44.361 ciudadanos en posesión de la licencia de pesca a los que se unen restantes pescadores con licencia interautonómica expedida por otra comunidad, calculándose en toral unos 65.000 ciudadanos en posesión de licencia de Pesca para los distintos tipos de escenarios (ríos, arroyos y embalses).

Por otro lado, en la Comunidad de Madrid existen un total de 22 tramos acotados o "cotos de pesca" entendidos como aquellos tramos y masas de aguas delimitadas, con especiales condiciones hidrobiológicas, donde el ejercicio deportivo de la pesca se desarrolla con fines exclusivamente recreativos, mediante un aprovechamiento ordenado que regule la presión de pesca de los mismos.

Por ello se hace necesaria una regulación ordenada de los escenarios, por ejemplo agrupando la pesca deportiva en tramos de extracción de ejemplares procedentes de repoblación, aminorando la posible presión de pesca sobre otros escenarios de mayor calidad medioambiental derivada de la alta demanda social de dicha actividad.

Por otro lado, en segundo lugar, la Comunidad de Madrid viene desarrollando desde el mes de noviembre de 2011, la gestión de las especies alóctonas, algunas de las cuales han provocado un debate técnico y social sobre su carácter nocivo o invasor. Entre ellas se encuentra la especie trucha arcoíris (Oncorhynchus mykiss W, 1972, Salmo gairdnieri-Richardson 1836) fue introducida por repoblación a finales del siglo con origen en Norteamérica y Centroeuropa existiendo constancia de su presencia histórica en Madrid en las cuencas de Lozoya, Manzanares, Guadarrama y Alberche.

Pues bien, se da la circunstancias de que la especie en cuestión ha variado su consideración desde la inclusión en un listado de especies exóticas potencialmente invasoras, posteriormente anulado, pasando por la suspensión cautelar de dicha norma por sentencias de la Sala de lo Contencioso- 
Administrativo del Tribunal Supremo o autorizaciones excepcionales para recintos antropizados o vinculados a actividades humanas en embalses.

Tras diversos avatares, la Sentencia 637/2016 de la Sala Tercera del Tribunal Supremo, de fecha 17 de junio de 2016, estimó parcialmente el recurso contencioso-administrativo contra el Real Decreto 630/2013, de 2 de agosto, por el que se regula el Catálogo español de especies exóticas invasoras, anulando parcialmente algunos extremos del mismo que afectan a la pesca.

Posteriormente, la Ley de $7 / 2018$, de 20 de julio, de modificación de la Ley 42/2007, de 13 de diciembre, del Patrimonio Natural y de la Biodiversidad trató de compatibilizar la lucha contra las especies exóticas invasoras con su aprovechamiento para la pesca, valorando sus beneficios sociales y económicos, en aquellas áreas que, al estar ocupa- das desde antiguo, su presencia no suponga un problema ambiental.

Concretamente se atribuye a las CCAA determinar las especies catalogadas que pueden ser objeto de aprovechamiento piscícola o cinegético, introducidas en el medio natural antes de la entrada en vigor de dicha ley, con el objeto de que se extiendan fuera de los límites de sus áreas de distribución, todo ello mediante la aprobación previa de los primeros instrumentos de planificación y gestión. No obstante, la Disposición transitoria segunda permite que, hasta que se aprueben los instrumentos normativos se pueda autorizar la práctica de la caza y de la pesca, en zonas delimitadas, de las especies que tengan relevancia social, y/o económica, en sus distintas modalidades, adoptando las debidas medidas tendentes a la salvaguarda del medio natural y del ecosistema donde se desarrollen.

La Resolución en cuestión autoriza, por todo ello, las sueltas con la especie trucha arcoíris (Oncorhynchus mykiss) exclusivamente en las masas de agua autorizadas antes de la entrada en vigor de dicha Ley 42/2007 y solo en aquellos tramos en que existía constancia de la presencia previa de la misma y no existía constancia de amenaza grave de afección sobre otras especies autóctonas, a saber:

- Embalse de La Jarosa*, en término municipal de Guadarrama. 
- Embalse de Navalmedio*, aporte del arroyo de Navalmedio en término municipal de Cercedilla.

- Embalse de Navacerrada*, en término municipal de Navacerrada

- Tramo II del coto de Molino de la Horcajada río Lozoya en término municipal del Lozoya del Valle. Canencia, Garganta de los Montes y Gargantilla.

- Tramo Il del coto del Río Manzanares en término municipal de Manzanares el Real,

- Tramo II del coto del río Aceña* en término municipal de Santa María de la Alameda

6. ORDEN 1501/2019, DE 18 DE JULIO, POR LA QUE SE MODIFICA LA ORDEN 2157/2013, DE 23 DE SEPTIEMBRE, DEL CONSEJERO DE MEDIO AMBIENTE Y ORDENACIÓN DEL TERRITORIO, POR LA QUE SE ESTABLECEN LAS BASES REGULADORAS PARA LA CONCESIÓN DE LAS AYUDAS PARA LA ADQUISICIÓN DE VEHÍCULOS EFICIENTES PARA USO DE AUTOTAXI

Esta Orden da unos datos muy relevadores de la problemática ambiental que generan los vehículos de autotaxi: "En la Comunidad de Madrid operan aproximadamente 16.000 vehículos autotaxi, que representan solo un 0,5 por 100 del total de vehículos matriculados, y sin embargo, son responsables de alrededor del 14 por 100 de las emisiones de dióxido de nitrógeno (NO2) de la región, por lo que las medidas de renovación tecnológica del sector son mucho más eficientes que las de renovación del conjunto del parque circulante, a la hora de disminuir las emisiones de $\mathrm{NO}_{2}$, que es uno de los contaminantes que registra superaciones de los objetivos de calidad establecidos por la Unión europea, principalmente en Madrid y su área metropolitana".

Pese a esta realidad, se apuesta desde la Comunidad de Madrid por continuar invirtiendo en este tipo de vehículos, actuando al margen de la problemática de las licencias VTC y sin una planificación previa de las necesidades del sector, incentivando el empleo de tecnologías menos contaminantes y sea renovada la flota actual de autotaxi. 
Dicha línea de actuación encaja, según la Consejería de Medio Ambiente y Ordenación del Territorio, en la nueva "Estrategia de calidad del aire y cambio climático de la Comunidad de Madrid 2013-2020. Plan Azul+", que contempla políticas de apoyo al vehículo eficiente, con el objetivo de contribuir a la mejora de la eficiencia energética y la reducción de las emisiones de $\mathrm{CO}_{2}$ y de contaminantes en las ciudades, al tiempo que se reduce la dependencia del petróleo y se favorece la utilización de fuentes de energía autóctonas.

Mediante la Orden de referencia, se modifican las bases reguladoras para la concesión de ayudas para la adquisición de vehículos eficientes de autotaxi, que están reguladas en la Orden 2157/2013, de 23 de septiembre.

No obstante, la modificación no conlleva efectos sustantivos relevantes desde la perspectiva medioambiental pues en realidad consiste en viabilizar las ayudas de los beneficiarios a través de entidades sin ánimo de lucro, un mecanismo algo a nuestro juicio sospechoso teniendo en cuenta el escenario mencionado de la dialéctica con las VTC.

Pero al margen de apreciaciones subjetivas no probadas, las bases establecen la figura del convenio de colaboración entre el órgano administrativo concedente y la entidad colaboradora, cuya selección se encuentra sujeta a procedimiento de concurrencia, tal y como establece el artículo 16.5 de la Ley $38 / 2003$, de 17 de noviembre, General de Subvenciones, en el que se prima como criterios los recursos humanos destinados a la gestión y pago de las ayudas.

\section{ACUERDO DE LA JUNTA DE GOBIERNO LOCAL DEL AYUNTAMIENTO DE MADRID DE FECHA 27 DE JUNIO DE 2019, POR EL QUE "PREVIA AVOCACIÓN DE COMPETENCIAS, SE ESTABLECE UN PERÍODO DE AVISO EN RELACIÓN CON EL ACCESO A LA ZONA DE BAJAS EMISIONES MADRID CENTRAL}

La Ordenanza de Movilidad Sostenible de la ciudad de Madrid, de 5 de octubre de 2018 (OMS), regula las denominadas Zonas de Bajas Emisiones (ZBE), que se definen conforme a su artículo 21 OMS como: "el ámbito territorial, conformado por el conjunto de vías públicas debidamente delimitadas que 
presenten continuidad geográfica, en que se implanten medidas especiales de regulación de acceso, circulación y estacionamiento de vehículos, para la reducción de las emisiones contaminantes procedentes del tráfico."

El artículo 23 OMS regula la ZBE Madrid Central, que a su vez fue desarrollada en lo relativo a su gestión mediante el Acuerdo de la Junta de Gobierno de la ciudad de Madrid de 5 de octubre de 2018. Dicha ZBE comenzó a funcionar el día 16 de marzo de 2019, tras un periodo de preaviso de dos meses previsto en el artículo 247 OMS.

Sin embargo, tras la celebración de las elecciones locales y la llegada de un nuevo equipo de gobierno de la Corporación municipal, con fecha 27 de junio de 2019, la Junta de Gobierno Local aprobó una Acuerdo por el que "previa avocación de competencias, se establece un período de aviso en relación con el acceso a la zona de bajas emisiones Madrid Central".

Dicho Acuerdo posee así un triple contenido:

1/. Avocar por la Junta de Gobierno Local las competencias para incoar, tramitar y resolver los expedientes sancionadores derivados los accesos no autorizados a la ZBE Madrid Central

2/. Poner en marcha una auditoría sobre el sistema automatizado de control de accesos a dicha Zona

3/. Establecer un periodo de preaviso a los conductores que accedan a Madrid Central de forma no autorizada, durante el cual no se impondrían sanciones, hasta que dicha auditoría no finalice

El Acuerdo fue publicado en el Boletín Oficial del Ayuntamiento de Madrid ํㅡㄴ 8429, y en el Boletín Oficial de la Comunidad Autónoma de Madrid no 154, ambos de la misma fecha de 1 de julio de 2019.

Contra ese Acuerdo de suspensión de la imposición de sanciones por accesos no permitidos a Madrid Central, se alzaron judicialmente las entidades ecologistas Greenpeace y Ecologistas en Acción, así como el grupo socialista municipal del Ayuntamiento de Madrid. Del resultado de dicha impugnación se da cuenta en la sección de jurisprudencia de este número de la Revista, si bien 
adelantamos que en la actualidad los procedimientos se encuentran pendientes de resolución final. 